\title{
BMJ Open Antenatal care booking within the first trimester of pregnancy and its associated factors among pregnant women residing in an urban area: a cross-sectional study in Debre Berhan town, Ethiopia
}

To cite: Kolola T, Morka W, Abdissa B. Antenatal care booking within the first trimester of pregnancy and its associated factors among pregnant women residing in an urban area: a crosssectional study in Debre Berhan town, Ethiopia. BMJ Open 2020;10:e032960. doi:10.1136/ bmjopen-2019-032960

- Prepublication history and additional material for this paper are available online. To view these files, please visit the journal online (http://dx.doi. org/10.1136/bmjopen-2019032960).

Received 19 July 2019 Revised 16 March 2020 Accepted 11 May 2020

A) Check for updates

(c) Author(s) (or their employer(s)) 2020. Re-use permitted under CC BY-NC. No commercial re-use. See rights and permissions. Published by BMJ.

${ }^{1}$ Department of Public Health, Ambo University, Ambo, Oromia, Ethiopia

${ }^{2}$ Department of Midwifery, Arsi University, Assela, Oromia,

Ethiopia

Correspondence to

Tufa Kolola;

tufabest@gmail.com

\section{ABSTRACT}

Objective This study aimed to assess antenatal care (ANC) booking within the first trimester of pregnancy and its associated factors among urban dwellers of pregnant women in Debre Berhan town, Ethiopia.

Design Cross-sectional.

Setting Public and private health facilities that provide ANC services in Debre Berhan town.

Outcome measure First ANC booking within the first trimester of pregnancy.

Participants Urban dwellers of pregnant women in Debre Berhan town $(n=384)$.

Results A total of 387 pregnant women in Debre Berhan town were selected for this study, of which 384 responded giving a response rate of $99.2 \%$. The proportion of pregnant women who had ANC booking within the first trimester of pregnancy was 156 (40.6\%; $95 \% \mathrm{Cl}$ : $35.8 \%$ to $45.6 \%)$. In the multivariable analysis, the odds of first ANC booking within the first trimester was higher among pregnant women who had secondary school (adjusted OR (AOR): 1.84; 95\% Cl: 1.10 to 3.19) and more than secondary level of education (AOR: $2.26 ; 95 \% \mathrm{Cl}: 1.27$ to 4.03 ) compared with those who had less than a secondary school level of education. Pregnant women who have any ill health with their current pregnancy (AOR: 1.99; 95\% Cl: 1.21 to 3.27) were more likely to start booking within the first trimester than their counterparts. The odds of ANC booking within the first trimester was threefold higher among women with knowledge of ANC (AOR: 3.05; $95 \% \mathrm{Cl}: 1.52$ to 6.11 ) compared with their counterparts. Conclusion First ANC booking within the first trimester was found to be low among urban dwellers of Debre Berhan town. Secondary school and more educational level, having ill health during early pregnancy and women's knowledge about ANC services were statistically associated with ANC booking within the first trimester of pregnancy. Therefore, improving ANC booking according to the WHO recommendation requires due attention. Further qualitative research exploring why early ANC booking remains low among urban dwellers is important to design intervention modalities.

\section{INTRODUCTION}

Poor maternal and newborn care remains a significant problem worldwide. ${ }^{1}{ }^{2}$
Strengths and limitations of this study

- This study used primary data for which missing or data incompleteness is minimal.

- This study was conducted among urban women, hence findings are not generalisable to other settings.

- Variables of interest like education of the partner, partner involvement, previous antenatal care use and stillbirth in previous pregnancy had significantly missing data and could not be included in the analysis.

Globally, an estimated 303000 women died from pregnancy-related causes, ${ }^{34}$ and about 2.6 million babies were stillborn in $2015 .^{5}$ Many of these adverse outcomes can be prevented by commencing ANC follow-up within the early pregnancy period. ${ }^{126}$ Within the continuum of care, antenatal care (ANC) provides a platform for critical healthcare functions including health promotion, prevention, screening and diagnosis of diseases. ${ }^{26-9}$ Implementing timely and appropriately, evidence-based interventions during ANC contact can improve maternal and fetal health. ${ }^{1011}$

ANC contact has been shown to improve positive pregnancy outcomes when the booking starts within the recommended pregnancy period and if the frequencies of contacts are adequate. Accordingly, WHO recommends pregnant women have to start their first ANC booking within the first 12 weeks of gestational age, with following contacts taking place at 20, 26, 30, 34, 36, 38 and 40 weeks of gestational age. ${ }^{7}$ When pregnant women start their ANC booking at an early stage of pregnancy, there might be more chances of getting treated from pregnancy-related ill health which negatively 
Table 1 Sociodemographic characteristics of the study participants, Debre Berhan town, Ethiopia, 2018

\begin{tabular}{lll}
\hline Variables & $\begin{array}{l}\text { Frequency } \\
(\mathbf{n = 3 8 4 )}\end{array}$ & Percentage \\
\hline Mother's age at birth (years) & & \\
$15-24$ & 115 & 30.0 \\
\hline $25-29$ & 164 & 42.7 \\
$\geq 30$ & 105 & 27.3 \\
\hline Marital status & & \\
\hline Married & 330 & 85.9 \\
\hline Unmarried & 54 & 14.1 \\
\hline
\end{tabular}

Religion

\begin{tabular}{|c|c|c|}
\hline Christian & 346 & 90.1 \\
\hline Muslim & 38 & 9.9 \\
\hline \multicolumn{3}{|l|}{ Education level } \\
\hline Less than secondary & 126 & 32.8 \\
\hline Secondary & 144 & 37.5 \\
\hline More than secondary & 114 & 29.7 \\
\hline \multicolumn{3}{|l|}{ Family size } \\
\hline $1-2$ & 165 & 43.0 \\
\hline $3-4$ & 171 & 44.5 \\
\hline$\geq 5$ & 48 & 12.5 \\
\hline \multicolumn{3}{|l|}{ Gravida } \\
\hline Primigravida & 184 & 47.9 \\
\hline Multigravida & 200 & 52.1 \\
\hline \multicolumn{3}{|l|}{ Parity } \\
\hline 0 & 204 & 53.1 \\
\hline$\geq 1$ & 180 & 46.9 \\
\hline \multicolumn{3}{|l|}{ Know ANC services } \\
\hline Yes & 314 & 81.8 \\
\hline No & 70 & 18.2 \\
\hline \multicolumn{3}{|l|}{ Pregnancy-related ill health } \\
\hline Yes & 273 & 71.1 \\
\hline No & 111 & 28.9 \\
\hline
\end{tabular}

ANC, antenatal care.

affects either the women or her baby. At ANC follow-up clinic, increasing maternal and fetal assessments to detect complications, improving support and communication between healthcare providers and pregnant women increases the likelihood of positive pregnancy outcomes. ${ }^{8}$

Ethiopia has implemented successive Health Sector Development Plans (HSDPs) following the Millennium Development Goals target. Maternal health is the major area where different efforts and contributions have been made from the beginning of HSDP with strong political will and commitment. ${ }^{12}$ As a result, Ethiopia has remarkably reduced pregnancy-related maternal mortality in the last two decades. ${ }^{13-15}$ Despite the appreciable progress the country has made, pregnancy-related maternal mortality

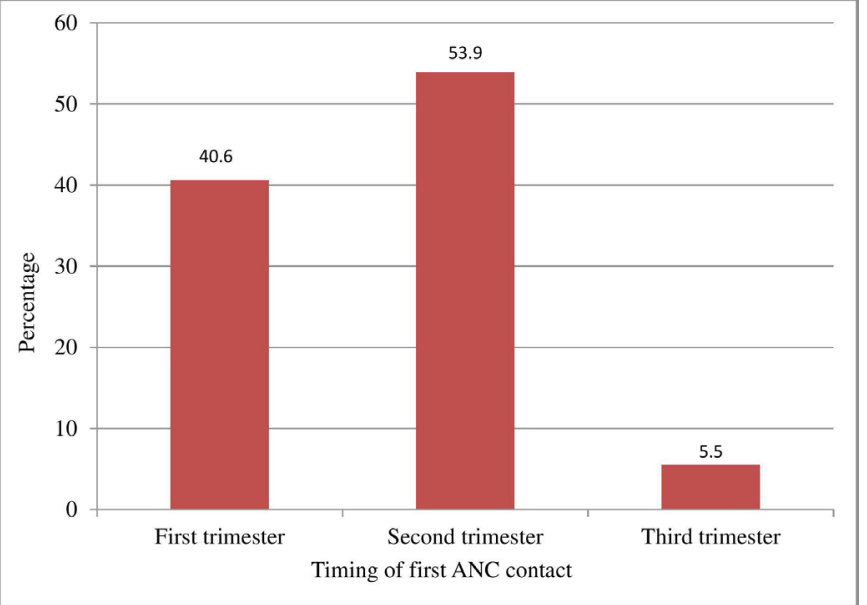

Figure 1 First ANC booking among urban dwellers of pregnant women in Debre Berhan town, Ethiopia, 2018. ANC, antenatal care.

remained one of a post-Millennium Development Goal priority agenda. To further speed the pace of progress in positive pregnancy outcomes, Ethiopia has emphasised strengthening the provision of ANC services at all levels in the health system. Improving the uptake of ANC service is one of the areas where due attention is given by the government. ${ }^{12}$ This, in fact, gradually improved ANC coverage, however; usage of ANC service at recommended gestational age varies by urban-rural dwellers in Ethiopia. ${ }^{16}$

Despite the privilege they have in terms of access to health facilities that provide ANC service, the majority of women residing in urban parts of Ethiopia do not use ANC service according to the WHO recommendation. ${ }^{17}$ For instance, the proportion of pregnant women who had their first ANC booking within the first trimester was found to be low. ${ }^{14}$ Previous studies have tried to assess the timing and factors associated with the first ANC booking among urban and rural dwellers combined. ${ }^{18} 19$ However, up-to-date information showing first ANC booking within the first trimester among urban residents was scanty including in North Shoa Zone of Amhara regional state. There was no previous study conducted on ANC booking within the first trimester among urban residents of pregnant women in Debre Berhan town so far. Therefore, this study aimed to assess first ANC booking within the first trimester of pregnancy and its associated factors among urban dwellers of pregnant women in Debre Berhan town, Ethiopia.

\section{METHODS}

\section{Study area and period}

This study was conducted in Debre Berhan town during April 2018. Debre Berhan town is located in North Shoa Zone of Amhara regional state which is about $130 \mathrm{~km}$ away from Addis Ababa, the capital city of Ethiopia. According to the 2007 Ethiopian census, this town has a total of 94829 population, of whom the reproductive age 
Table 2 Bivariate and multivariable logistic regression analyses for first ANC booking within the first trimester of pregnancy among urban dwellers of pregnant women in Debre Berhan town, Ethiopia, 2018

\begin{tabular}{|c|c|c|c|c|}
\hline \multirow[b]{2}{*}{ Variables } & \multicolumn{2}{|c|}{$\begin{array}{c}\text { First ANC booking within the first } \\
\text { trimester }\end{array}$} & \multicolumn{2}{|c|}{ OR $(95 \% \mathrm{Cl})$} \\
\hline & Yes & No & Unadjusted & Adjusted \\
\hline \multicolumn{5}{|c|}{ Mother's age at birth (years) } \\
\hline $15-24$ & 43 & 72 & 1.25 (0.72 to 2.18$)$ & $0.89(0.44$ to 1.78$)$ \\
\hline $25-29$ & 79 & 85 & $1.94(1.16 \text { to } 3.24)^{*}$ & 1.32 (0.74 to 2.36$)$ \\
\hline$\geq 30$ & 34 & 71 & 1 & 1 \\
\hline \multicolumn{5}{|l|}{ Marital status } \\
\hline Married & 140 & 190 & 1.75 (0.94 to 3.26$)$ & \\
\hline Unmarried & 16 & 38 & 1 & \\
\hline \multicolumn{5}{|l|}{ Education level } \\
\hline Less than secondary & 32 & 94 & 1 & 1 \\
\hline Secondary & 67 & 77 & $2.56(1.52 \text { to } 4.29)^{\star}$ & $1.84(1.10 \text { to } 3.19)^{\star}$ \\
\hline More than secondary & 57 & 57 & $2.94(1.70 \text { to } 5.06)^{\star}$ & $2.26(1.27 \text { to } 4.03)^{\star}$ \\
\hline \multicolumn{5}{|l|}{ Family size } \\
\hline $1-2$ & 75 & 90 & $2.24(1.11 \text { to } 4.55)^{\star}$ & 1.71 (0.73 to 3.98$)$ \\
\hline $3-4$ & 68 & 103 & $1.77(0.88 \text { to } 3.60)^{\star}$ & 1.35 (0.63 to 2.89$)$ \\
\hline$\geq 5$ & 13 & 35 & 1 & 1 \\
\hline \multicolumn{5}{|l|}{ Gravida } \\
\hline Primigravida & 84 & 100 & 1.49 (0.99 to 2.50$)$ & \\
\hline Multigravida & 72 & 128 & 1 & \\
\hline \multicolumn{5}{|l|}{ Parity } \\
\hline 0 & 93 & 111 & $1.56(1.03 \text { to } 2.35)^{\star}$ & 1.46 (0.95 to 2.25$)$ \\
\hline$\geq 1$ & 63 & 117 & 1 & 1 \\
\hline \multicolumn{5}{|l|}{ Know ANC services } \\
\hline Yes & 144 & 170 & $4.09(2.12 \text { to } 7.92)^{\star}$ & 3.05 (1.52 to 6.11$)$ \\
\hline No & 12 & 58 & 1 & 1 \\
\hline \multicolumn{5}{|c|}{ Pregnancy-related ill health } \\
\hline Yes & 124 & 149 & $2.06(1.28 \text { to } 3.30)^{\star}$ & $1.99(1.21 \text { to } 3.27)^{\star}$ \\
\hline No & 32 & 79 & 1 & 1 \\
\hline
\end{tabular}

*Statistically significant at $\mathrm{p}<0.05$.

ANC, antenatal care.

group (15-49 years) is 21972 . There are six health facilities offering ANC services in Debre Berhan town during the study period. These include one referral hospital, one general hospital, three health centres and one medium clinic, of which, the referral hospital and health centres are public health facilities. In our study, we included all health facilities within the town which provide ANC services. All pregnant women residing in this town and aged from 15 to 49 years were considered eligible for this study.

\section{Study design and sample}

A cross-sectional study was conducted among 387 urban dwellers of pregnant women attending antenatal clinics at the health facilities of Debre Berhan town from 1 to 24 April 2018. The sample size was determined using a single population proportion formula by considering a $95 \%$ confidence level, $5 \%$ margin of error, the proportion of timely ANC booking which was $35.4 \%{ }^{20}$ and a $10 \%$ non-response rate.

A systematic sampling technique was used for sample selection by considering their order of ANC attendance as a sampling frame. First, the desired sampling interval was determined based on total ANC attendants a month before the actual data collection period $(834 / 387=2)$. The starting unit was chosen at random from the units corresponding to the first sampling interval and the subsequent units were then selected at this fixed interval. In the same way, another random start was chosen using the same sampling interval on the next day data collection, and this process was repeated until the last day of 
data collection. We used the same sampling interval to ensure probability proportionate to size selection from each health facility. Besides, we included all health facilities within the town which provide ANC services. Therefore, the sample size is representative.

\section{Patient and public involvement}

No patient involved.

\section{Data collection tools and procedure}

Data were collected using a structured questionnaire (online supplementary S1 appendix) adopted from the Ethiopian Demographic and Health Survey ${ }^{14}$ and a previous study. ${ }^{20}$ The questionnaire was designed to gather data on sociodemographic characteristics of the study participants, first ANC booking within the first trimester of pregnancy and factors associated with first ANC booking among pregnant women. The questionnaire was pretested on $5 \%$ of the sample size $(n=20)$ in Keyit Health Center, one of the nearby health centre of Debre Berhan town. Based on the result of the pretest, amendments were made on some items of the questionnaire before actual data collection.

The data collectors who are fluent in the local language (in Amharic) and who know the culture of that community were recruited. They were provided training by investigators to familiarise themselves with the data collection tools and the procedure to be followed when collecting data. They are diploma Midwives who had experience in data collection. In short, six data collectors held face-toface exit interviews with study participants.

\section{Study variables}

The dependent variable of interest in this study was a binary response of whether a woman initiated the first ANC booking in the first trimester of pregnancy (yes/ no), whereas the independent variables were: mother's age at birth (15-24, 25-29 and $\geq 30$ years); marital status (married and unmarried); religion (Christian and Muslim); education level (less than secondary, secondary school and more than secondary); family size (1-2, 3-4 and $\geq 5$ ); gravida (primigravida and multigravida); parity ( 0 and $\geq 1)$; previous ANC use (yes/no); know ANC services (yes/no); stillbirth in a previous pregnancy (yes/ no) and history of current pregnancy-related ill health (yes/no).

\section{Data analysis}

Data entry and cleaning were carried out using Epi Info 7 and analysed by SPSS V.21. Descriptive analysis was done to determine the proportion of first ANC bookings within the first trimester of pregnancy. Bivariate and multivariable analyses were done using a binary logistic regression model to identify factors associated with the first ANC booking within the first trimester. Bivariate analysis was done first and then followed by multivariable analysis. Backward elimination method was used and variables significantly associated with first ANC booking within the first trimester of pregnancy, at $p$ value $<0.05$ in the bivariate analysis, were entered into the multivariable model. Adjusted ORs (AORs) with a 95\% CI were calculated for all variables taken to the multivariable model.

\section{RESULTS}

Sociodemographic characteristics of the study participants

A total of 387 pregnant women in Debre Berhan town were selected for this study, of which 384 responded giving a response rate of $99.2 \%$. One hundred and sixtyfour $(42.7 \%)$ of them belonged to the 24-29 years age group. A larger proportion of the study participants, $346(90.1 \%)$, were Christian followers by religion. About $144(37.5 \%)$ and $114(29.7 \%)$ of the participants had secondary and more than secondary level of education, respectively. Slightly more than half of the study participants were multigravida (52.1\%) and nulliparous (53.1\%) (table 1).

\section{First ANC booking within the first trimester of pregnancy}

In this study, the proportion of pregnant women who had their first ANC booking within the recommended time, that is, within the first trimester, was $156(40.6 \%$; $95 \%$ CI: $35.8 \%$ to $45.6 \%)$. Slightly more than half of the participants, 207 (53.9\%; 95\% CI: 48.9\% to 58.8\%), started their ANC booking during the second trimester and about 21 (5.5\%; 95\% CI: $3.5 \%$ to $8.1 \%$ ) of the participants booked during the third trimester (figure 1).

Factors associated with first ANC booking within the first trimester of pregnancy

In the multivariable analysis, mother's education level was significantly associated with first ANC booking within the first trimester of pregnancy. The odds of first ANC booking within the first trimester was higher among pregnant women who attended secondary school (AOR: 1.84; 95\% CI: 1.10 to 3.19 ) and more than secondary level of education (AOR: 2.26; 95\% CI: 1.27 to 4.03). Pregnant women who have pregnancy-related ill health (AOR: 1.99; $95 \%$ CI: 1.21 to 3.27 ) were more likely to start their first booking within the first trimester than their counterparts. The result also indicated that the odds of first ANC booking within the first trimester of pregnancy were threefold higher among women with knowledge of ANC (AOR: 3.05; 95\% CI: 1.52 to 6.11 ) compared with their counterparts. Mother's age at birth, family size and parity showed no significant association following adjustment for other variables (table 2).

\section{DISCUSSION}

The new, 2016, WHO ANC model highlights that a woman's 'ANC contact' with healthcare providers should be more than a simple 'visit' but should be an opportunity for good quality care. The new model recommends pregnant women to have their first contact within the first 12 weeks' gestation. ${ }^{78}$ In this study, however, only $40.6 \%$ of pregnant women had their first ANC booking 
within the first trimester which was higher compared with what has been reported by previous studies in Ethiopia. ${ }^{19-22}$ This difference could be that we conducted this study among urban residents, whereas study participants were from both urban and rural residents in the previous studies. Similarly, the finding from this study was higher compared with finding from a study done among urban dwellers of Jimma, Serbo and Agaro. ${ }^{23}$ The reason for the observed differences might be that a study from Jimma, Serbo and Agaro was conducted a long time ago and its finding does not represent the current realities.

In contrast, the finding in this study was lower compared with the findings from six Mesoamerican countries (ie, from Guatemala, Honduras, Mexico, Nicaragua, Panama and El Salvador). Among women who attended at least one ANC, $73 \%$ had their first contact in the first trimester, from a low of $63 \%$ in Panama and Guatemala to a high of $82 \%$ in El Salvador. ${ }^{24}$ This difference might be due to the socioeconomic differences between our study settings and Mesoamerican countries. The difference could also be due to the discrepancy in pregnancy uncertainties between the countries.

The multivariable analysis of this study shows that the higher the educational level of pregnant women, the earlier they start ANC booking within the first trimester. This is consistent with the finding from a study done among urban residents of pregnant women in Zambia. ${ }^{25}$ Likewise, postprimary education level was associated with an increased odds of first ANC contact in the first trimester in Guatemala, Mexico and Nicaragua. ${ }^{24}$ This could be due to the fact that mothers' knowledge regarding the benefits of early ANC booking increases with their educational level. This, in short, suggests that women's decision making for early ANC contact by far increases as their education advances.

This study also indicated that the odds of starting first ANC contact within the first trimester of pregnancy was twofold more likely among pregnant women who have any health problem with their current pregnancy than their counterparts. This finding is similar to the study done in Kampala which showed that more than half of the pregnant mothers did not have any problem with their current pregnancy and so they saw no reason to come early for ANC. ${ }^{26}$ The possible explanation might be pregnant women are more concerned to have ANC contact when they feel discomfort with their pregnancy during the first few weeks of gestation.

This study has several limitations. One limitation is that this study is conducted among urban women, hence, findings are not generalisable to other settings. Another limitation is that some variables of interest like educational level of the partner, partner involvement, previous ANC use and stillbirth in previous pregnancy had significantly missing data and could not be included in the analysis.

In conclusion, this study showed that less than half of urban dwellers pregnant women had their first ANC booking within the first trimester of pregnancy in Debre
Berhan town. Being attended postprimary education, having health problems during early pregnancy and women's knowledge regarding ANC services were associated with increased odds of first ANC contact in the first trimester. Qualitative research exploring why early ANC contact remains low among urban dwellers is important to design intervention modalities.

Acknowledgements The authors would like to acknowledge Debre Berhan University for funding this study. Authors are also grateful to study subjects for giving their time and information.

Contributors TK: conception of the idea, study design, facilitation of data collection, data analysis and drafted the manuscript. WM: conception of the idea, study design, facilitation of data collection and revision of the manuscript. BA: study design, facilitation of data collection and revision of the manuscript. All authors read and approved the final manuscript.

Funding This study was funded by Debre Berhan University.

Competing interests None declared.

Patient consent for publication Not required.

Ethics approval The proposal of this study was approved by the Ethics Review Committee of College of Health Sciences, Debre Berhan University (ref: RCSVP/358/07-11/63). Then the ethical clearance letter was obtained from the college and submitted to the zonal health bureau for permission to undertake the study. Verbal informed consent was obtained from all study participants before the interview. Moreover, study participants were not identified for confidentiality reasons.

Provenance and peer review Not commissioned; externally peer reviewed.

Data availability statement Data are available upon reasonable request. Data set underlying the findings can be found by requesting Tufa Kolola:tufabest@gmail. com.

Open access This is an open access article distributed in accordance with the Creative Commons Attribution Non Commercial (CC BY-NC 4.0) license, which permits others to distribute, remix, adapt, build upon this work non-commercially, and license their derivative works on different terms, provided the original work is properly cited, appropriate credit is given, any changes made indicated, and the use is non-commercial. See: http://creativecommons.org/licenses/by-nc/4.0/.

\section{ORCID iDs}

Tufa Kolola http://orcid.org/0000-0003-0339-8176

Wogene Morka http://orcid.org/0000-0001-5458-1507

\section{REFERENCES}

1 UNICEF. The state of the world's children. maternal and newborn health, 2009. Available: https://www.unicef.org/sowc09/

2 Lassi ZS, Salam RA, Das JK, et al. Essential interventions for maternal, newborn and child health: background and methodology. Reprod Health 2014;11:S1.

3 Alkema L, Chou D, Hogan D, et al. Global, regional, and national levels and trends in maternal mortality between 1990 and 2015, with scenario-based projections to 2030: a systematic analysis by the un maternal mortality estimation inter-agency group. Lancet 2016;387:462-74.

4 World Health Organization. Trends in Maternal Mortality : 1990 to 2015. Geneva, Switzerland, 2015.

5 Blencowe H, Cousens S, Jassir FB, et al. National, regional, and worldwide estimates of stillbirth rates in 2015, with trends from 2000: a systematic analysis. Lancet Glob Health 2016;4:e98-108.

6 Lunze K, Higgins-Steele A, Simen-Kapeu A, et al. Innovative approaches for improving maternal and newborn health--A landscape analysis. BMC Pregnancy Childbirth 2015;15:337.

7 World Health Organization. WHO recommendations on antenatal care for a positive pregnancy experience, 2016. Available: https:// apps.who.int/iris/bitstream/10665/250796/./9789241549912-eng.pdf

8 Tunçalp Ö, Pena-Rosas JP, Lawrie T, et al. WHO recommendations on antenatal care for a positive pregnancy experience-going beyond survival. BJOG 2017;124:860-2.

9 National Institute for Health and Clinical Excellence. Antenatal care: routine care for the healthy pregnant woman, 2010. Available: https:// www.ncbi.nlm.nih.gov/pubmed/21370514 
10 World Health Organization. WHO statement on antenatal care, 2011. Available: whqlibdoc.who.int/hq/2011/WHO_RHR_11.12_eng.pdf

11 Saad-Haddad G, DeJong J, Terreri N, et al. Patterns and determinants of antenatal care utilization: analysis of national survey data in seven countdown countries. J Glob Health 2016;6:010404.

12 Federal Ministry of Health. Health ector transformation plan: 2015/16 - 2019/20, 2015. Available: https://www.globalfinancingfacility.org/./ Ethiopia-health-system- transformation-plan.pdf

13 World Health Organization. Success factors for Women's and children's health: policy and programme highlights from 10 fast-track countries, 2014. Available: https://www.who.int/pmnch/./success_ factors highlights.pdf

14 Central Statistical Agency. Ethiopia demographic and health survey 2016, 2017. Available: https://dhsprogram.com/pubs/pdf/FR328/ FR328.pdf

15 Federal Ministry of Health. Policy and practice -information for action. Q Heal Bull 2014;6:1-51

16 Mezmur M, Navaneetham K, Letamo G, et al. Socioeconomic inequalities in the uptake of maternal healthcare services in Ethiopia. BMC Health Serv Res 2017;17:1-11.

17 Gizaw Met al. Maternal health service utilization in urban slums of selected towns in Ethiopia: qualitative study. Ethiop J Heal Dev 2017;31:96-102.

18 Zegeye AM, Bitew BD, Koye DN. Prevalence and determinants of early antenatal care visit among pregnant women attending antenatal care in Debre Berhan health institutions, central Ethiopia. Afr J Reprod Health 2013;17:130-6.
19 Gebremeskel F, Dibaba Y, Admassu B. Timing of first antenatal care attendance and associated factors among pregnant women in Arba Minch town and Arba Minch district, Gamo Gofa zone, South Ethiopia. J Environ Public Health 2015;2015:1-7.

20 Gudayu TW, Woldeyohannes SM, Abdo AA. Timing and factors associated with first antenatal care Booking among pregnant mothers in Gondar town; North West Ethiopia. BMC Pregnancy Childbirth 2014;14:287.

21 Abosse Z, Woldie M, Ololo S. Factors influencing antenatal care service utilization in hadiya zone. Ethiop J Health Sci 2010;20:75-82.

22 Tekelab T, Berhanu B. Factors associated with late initiation of antenatal care among pregnant women attending antenatal clinic at public health centers in Kembata Tembaro zone, southern Ethiopia. Sci Technol Arts Res J 2014;3:108-15.

23 Villadsen SF, Tersbøl BP, Negussie D, et al. Antenatal care strengthening in Jimma, Ethiopia: a mixed-method needs assessment. J Environ Public Health 2014;2014:1-10.

24 Dansereau E, McNellan CR, Gagnier MC, et al. Coverage and timing of antenatal care among poor women in 6 Mesoamerican countries. BMC Pregnancy Childbirth 2016;16:234.

25 Sinyange N, Sitali L, Jacobs C, et al. Factors associated with late antenatal care booking: population based observations from the 2007 Zambia demographic and health survey. Pan Afr Med $J$ 2016;25:1-11.

26 Kisuule I, Kaye DK, Najjuka F, et al. Timing and reasons for coming late for the first antenatal care visit by pregnant women at Mulago Hospital, Kampala Uganda. BMC Pregnancy Childbirth 2013;13:121. 